\title{
Breast Medical Oncology in the Era of COVID-19 Pandemic
}

\author{
Rania Chacha, Said Afqir
}

Department of Medical Oncology, Mohamed VI University Hospital, Oujda, Morocco.

\begin{abstract}
The coronavirus disease 2019 (COVID-19) has spread over the majority of countries and it was declared as a pandemic on March $11^{\text {th }}, 2020$ by The World Health Organization. The number of infected people worldwide is still in progress. Patients diagnosed with cancer are a fragile population because of their chronic disease and related treatments, and therefore, requiring a particular management. Several international initiatives from scientific societies and working groups have provided updated guidance to improve the medical treatments of breast cancer. These recommendations aimed to mitigate the negative effects of this outbreak on prevention, diagnosis, and treatments. Notably, this should be used for prioritizing the various aspects of cancer care particularly breast cancer patients' safety. In this editorial, we shortly review the current guidelines for breast cancer management during this pandemic and illustrate the available treatments that should be prioritized. We believe this paper will be useful for young oncologists and physicians dealing with this emerging life-threatening disease.
\end{abstract}

Keywords: COVID-19- breast cancer- management

Asian Pac J Cancer Care, 5 (Suppl 1), 187-190

\section{COVID-19 Pandemic: Brief Overview}

Because of the rapidly progressing COVID-19 outbreak, it was considered as a public health emergency globally. The virus associated with this disease called severe acute respiratory syndrome coronavirus 2 (SARS-CoV-2) was first detected in Wuhan, China in December 2019 [1]. It has similarities with SARS-CoV-1 which was the cause of SARS pandemic in 2002 [1]. Historically, Tyrell and Bynoe have first identified this family of coronaviruses in 1966 [2]. There are three different variants of this virus $\mathrm{A}, \mathrm{B}$ and $\mathrm{C}$, with some differences in their structural amino acid sequences [3]. This virus belongs to the Coronavirus (CoV) family which are RNA viruses [4]. SARS-CoV-2 particles form like crown which is the origin of the name (coronavirus) [5]. Its size varies from $65-125 \mathrm{~nm}$ in diameter [6]. Moreover, there are low and high pathogenic CoVs [3]. The bats constitute its primary reservoir and its transmission between humans is very quick [6]. Its detection is based on quantitative real-time RT-PCR (RT-qPCR) which is very sensitive [7]. In addition, specific antibody detection may also be used for diagnosis [7]. The primary route of transmission is from animals to humans, however; transmission from humans to humans like in influenza
Submission Date: 07/05/2020Ａcceptance Date: 08/04/2020

and rhinoviruses cases requires isolation as the best strategy to reduce the epidemic evolution [4]. Clinically, COVID-19 patients can be asymptomatic, in which $20 \%$ may develop severe conditions requiring hospitalization [3]. Fever, cough, asthenia are the principal symptoms [5]. Moreover, diarrhea can be present rarely in some patients [5]. A number of chronic comorbidities such as cancer, hypertension, diabetes, obesity, may be associated with worse outcomes [3]. Radiologically, computed tomography shows peripheral distribution of frosted and reticular opacities without pleural effusion, Ground-glass opacity, consolidation and air bronchi sign, Paving stone sign, fibrous lesions, and vascular thickening [4]. Patients treated for cancer are vulnerable population; they can be infected with a high risk of death during COVID-19 [8] as compared to the general population [1]. A recent cohort demonstrated that patients with cancer have an increased risk of complications [9]. Thus, prioritization of management and healthcare services should be urgently implemented. Patients with cancer under treatments that may impact their survival outcomes should be continued despite the current pandemic [10]. Several updates in international guidelines for chemotherapy,

\section{Corresponding Author:}

Dr. Rania Chacha

Department of Medical Oncology, Mohamed VI University Hospital, Oujda, Morocco.

Email: chacharania890@gmail.com 
immunotherapy [9], radiation therapy, etc, have been implemented as an urgent response from the oncology community. In the following section, we revisit breast medical oncology during COVID-19.

\section{Global Response of the Oncological Societies}

Cancer patients are facing unprecedented circumstances during the COVID-19 pandemic. The response of the oncologists and the oncology community to the COVID-19 outbreak has rapidly changed the management of breast cancer in some settings [11]. Notable changes were observed worldwide in reorganization of health care management and internal procedures without significant impact neither on radiotherapy or chemotherapy plans, nor histopathological analyses. However, the cancer diagnostic activity was drop considerably [12]. A number of independent initiatives and international oncology societies have provided novel guidance to improve the management of breast cancer during this pandemic. A quick search on Pubmed/Medline database uncovered more than 50 publications on this hot topic demonstrating a rapid response of the oncologists to provide adapted guidelines and expert commentaries and therefore, better manage breast cancer. In this section, we revisit this response to this pandemic based on a selected set of papers.

The ESMO breast cancer recommendations were rapidly adapted to the COVID-19 occurrence to accurately respond to this clinical urgency [13]. Briefly, for early breast cancer, the use of neoadjuvant chemotherapy (NACT) and adjuvant treatments for triple-negative histotype should be considered as high priorities. Similarly, these chemotherapeutic regimens combined to targeted agents for HER-2 positive patients should also be prioritized. Medical oncologists have to follow the existing international guidelines for NACT and adjuvant endocrine therapy ( \pm chemotherapy) for high-risk ER-positive/HER2-negative tumors. The ESMO recommendations on NACT also stated the importance of the completion of already initiated treatments with or without anti-HER2 therapy as a high priority. Moreover, adjuvant capecitabine in triple-negative breast cancer patients with high-risk as well as trastuzumab emtansine in HER2-positive patients with high risk breast cancer should be continued. In the context of clinical trials, treatments should be continued with the possibility of adapting the procedures without affecting the patients' safety and study conduct. For medium priorities, stage I breast cancer in postmenopausal patients with low to intermediate grade tumors and lobular histology can benefit from endocrine therapy first and delay their surgery. In addition, endocrine therapy alone in patients that have low risk genomic signatures/scores is preferred. Postponement of ongoing adjuvant trastuzumab alone by 6 to 8 weeks in patients at high risk of COVID-19 related complications is recommended. Other statements including delay of a) follow-up imaging, b) restaging, c) echo(electro) cardiograms, and d) bone density scans in clinically asymptomatic patients or in those demonstrating clinical response in the neoadjuvant setting are considered a low priorities by these guidelines. In the metastatic setting, the high priorities are given to patients with visceral crisis, those candidates to early lines of chemotherapy, endocrine and targeted therapy, as well as immune-checkpoint blockade that considered as likely to improve survival outcomes in the metastatic disease. Of note, pertuzumab/ trastuzumab combined to chemotherapy in HER2 positive patients should be considered as a high priority. The combination of endocrine agents with CDK4-6 blockers in ER-positive/HER2-negative breast cancer patients and chemotherapy with atezolizumab in PD-L1 positive triple-negative breast cancer should be discussed in a multidisciplinary tumor board teams. A similar previous statement regarding the patients enrolled in clinical trials is mentioned. Medium priorities encompass second, third, and beyond third-lines of therapy when they improve outcomes. It is important to avoid or delay the addition of mTOR/PI3KCA blockers to endocrine treatments, mainly in elderly patients with comorbidities. Based on a prospective registry analysis, COVID-19 mortality in breast cancer is associated with comorbidities rather than the use of radiation or systemic anticancer therapies [14]. Therefore, attention should be paid to the presence of comorbidities for patients' risk estimation and stratification particularly in the elderly population [15]. The discussion of inclusion in clinical trials with breast cancer patients that may provide benefits is also a medium priority. Importantly, the use of bone modifying agents such as zolndronic acid that are not an urgent need, principally for hypercalcemia and pain control is a low priority and can be given once every three months. Additional procedures for follow up such as imaging can be delayed similarly to the early breast cancer setting or performed at expanded intervals (for additional review see [13-16].

The COVID-19 Pandemic Breast cancer Consortium lead by several American cancer societies has also provided support to the oncology physicians by a number of recommendations [17]. Priorities were categorized into three levels $\mathrm{A}, \mathrm{B}$, and $\mathrm{C}$ according to the current appraisal of the evidence and importantly, the severity of cancer patient's conditions. The expert panel considers a priority A when oncologic emergencies such as febrile neutropenia or symptomatic pleural effusion are diagnosed. A necessary management should be initiated according to the priority A recommending urgent interventions. In newly diagnosed breast cancer, a revision of multidisciplinary management plans is recommended to protect patients considered as priority B, for which they don't have immediate life-threatening disease but requiring no delays for their management. NACT has profound impact for all breast cancers and may delay surgical interventions. Patients with ER positive and HER2-negative tumors may benefit from delays in surgery without clinical compromise (priority B). This category of patients may be treated with neoadjuvant endocrine therapy for 6 months to one year. In case of stage 1 or limited stage 2 breast cancer as well as low to intermediate grade tumors, lobular histology, or low recurrence score, endocrine therapy alone is recommended as NACT or adjuvant regimens are not 
substantially beneficial in these settings. Those breast cancer patients (all subtypes) that already started NACT or adjuvant therapy should complete their treatments (priority B). Modifications and regimens shortening may be proposed. For example, sequential treatments based on monotherapy may reduce the risk of complications without reducing its efficacy. To minimize the risk of neutropenia and corticosteroids use, NACT or adjuvant therapy based on chemotherapy combined to trastuzumab can be replaced with ado-trastuzumab emtansine ( \pm pertuzumab). Importantly, the regimens using adjuvant trastuzumab can be shortened from one year to 6 months in selected patients. In advanced/metastatic breast cancer, adjustments in doses and schedules of systemic therapy may be recommended to reduce patients' visits, biological analyses, and management of adverse events. Restaging based on imaging can be differed in those patients that didn't show signs of cancer progression. In addition, HER 2 breast cancer patients with more than two years of disease control and minimal disease control with trastuzumab-based therapy may consider maintenance therapy interruption. Reduction of doses of agents with increased risk of toxicities such as CDK4/6 inhibitors in ER-positive metastatic breast cancer may be a solution for these adverse events requiring clinic visits. Endocrine therapy is recommended for patients with ER positive ductal carcinoma in situ for 6 months prior to surgery (priority C).

In conclusion, G-CSF growth factors use is recommended to prevent neutropenia secondary to chemotherapy. Moreover, oral chemotherapy is preferred and managing the toxicities via phone- and telemedicinebased are recommended for surveillance and routine follow-up except in cases of emergencies. Dose and schedule adaptation are also recommended when necessary. Chemotherapy infusions can be proceeded as usual. Importantly, management of metastatic breast cancer should be prioritized and the chemotherapeutic protocols can be used as usual [18]. In addition, because of the risk of immune-suppression associated to some drugs such as mTOR or PI3KCA inhibitors (everolimus) and dexamethasone, these agents should be avoided.

\section{Conflicts of interest}

None

\section{Funding \\ None}

\section{References}

1. Al-Shamsi HO, Alhazzani W, Alhuraiji A, Coomes EA, Chemaly RF, Almuhanna M, Wolff RA, Ibrahim NK, Chua ML, Hotte SJ, Meyers BM, Elfiki T, Curigliano G, Eng C, Grothey A, Xie C. A Practical Approach to the Management of Cancer Patients During the Novel Coronavirus Disease 2019 ( COVID -19) Pandemic: An International Collaborative Group. The Oncologist. 202004 27;25(6). https://doi.org/10.1634/theoncologist.2020-0213

2. Gosain R, Abdou Y, Singh A, Rana N, Puzanov I, Ernstoff MS. COVID-19 and Cancer: a Comprehensive Review. Current
Oncology Reports. 2020 05;22(5). https://doi.org/10.1007/ s11912-020-00934-7

3. Azkur AK, Akdis M, Azkur D, Sokolowska M, Veen W, Brüggen M, O’Mahony L, Gao Y, Nadeau K, Akdis CA. Immune response to SARS-CoV-2 and mechanisms of immunopathological changes in COVID-19. Allergy. 2020 07;75(7):1564-1581. https://doi.org/10.1111/all.14364

4. Gligorov J, Bachelot T, Pierga J, Antoine E, Balleyguier C, Barranger E, Belkacemi Y, Bonnefoi H, Bidard F, Ceugnart L, Classe J, Cottu P, Coutant C, Cutuli B, Dalenc F, Darai E, Dieras V, Dohollou N, Giacchetti S, Goncalves A, Hardy-Bessard A, Houvenaeghel G, Jacquin J, Jacot W, Levy C, Mathelin C, Nisand I, Petit T, Petit T, Poncelet E, Rivera S, Rouzier R, Salmon R, Scotté F, Spano J, Uzan C, Zelek L, Spielmann M, Penault-Llorca F, Namer M, Delaloge S. COVID-19 et personnes suivies pour un cancer du sein : recommandations françaises pour la pratique clinique de Nice-St Paul de Vence, en collaboration avec le Collège Nationale des Gynécologues et Obstétriciens Français (CNGOF), la Société d'Imagerie de la Femme (SIFEM), la Société Française de Chirurgie Oncologique (SFCO), la Société Française de Sénologie et Pathologie Mammaire (SFSPM) et le French Breast Cancer Intergroup-UNICANCER (UCBG). Bulletin du Cancer. 2020 05;107(5):528-537. https://doi.org/10.1016/j. bulcan.2020.03.008

5. Chen $\mathrm{H}, \mathrm{Ai} \mathrm{L}, \mathrm{Lu} \mathrm{H}, \mathrm{Li} \mathrm{H}$. Clinical and imaging features of COVID-19. Radiology of Infectious Diseases. 2020 04; https://doi.org/10.1016/j.jrid.2020.04.003

6. Shereen MA, Khan S, Kazmi A, Bashir N, Siddique R. COVID-19 infection: Origin, transmission, and characteristics of human coronaviruses. Journal of Advanced Research. 2020 07;24:91-98. https://doi.org/10.1016/j. jare.2020.03.005

7. Li C, Ren L. Recent progress on the diagnosis of 2019 Novel Coronavirus. Transboundary and Emerging Diseases. 2020 05 31;67(4):1485-1491. https://doi.org/10.1111/tbed.13620

8. Mauri D, Kamposioras K, Tolia M, Alongi F, Tzachanis D, Petricevic B, Chilingirova N, Mihaylova I, Tsakiridis T, Salembier C, Papadimitriou K, De Mello RA, Amaral GA, Neves NM, Dias R, Yi J, Dedic Plavetic N, Antunac K, Kountourakis P, Kopecky J, Christopoulos C, Pavese I, Corradini S, Zarkavelis G, Papadaki A, Gkantaifi A, Theodorelos V, Capizzello A, Lovey J, Cellini F, Dambrosio M, Takeuchi D, Saraireh HH, Røtterud R, Chojnacka M, Papachristos A, Djan I, Popovic L, Grašic Kuhar C, Strojan P, Hindi N, Valachis A, Åhs M, De Bari B, Santa Cruz O, Hameed H, Takeuchi E, Apostolidis K, Cardone A, Gono $\mathrm{P}$, Filis P. Summary of international recommendations in 23 languages for patients with cancer during the COVID-19 pandemic. The Lancet Oncology. 2020 06;21(6):759-760. https://doi.org/10.1016/s1470-2045(20)30278-3

9. Moujaess E, Kourie HR, Ghosn M. Cancer patients and research during COVID-19 pandemic: A systematic review of current evidence. Critical Reviews in Oncology/ Hematology. 2020 06;150:102972. https://doi.org/10.1016/j. critrevonc.2020.102972

10. Saini KS, de las Heras B, de Castro J, Venkitaraman R, Poelman M, Srinivasan G, Saini ML, Verma S, Leone M, Aftimos P, Curigliano G. Effect of the COVID-19 pandemic on cancer treatment and research. The Lancet Haematology. 2020 06;7(6):e432-e435. https://doi.org/10.1016/s23523026(20)30123-x

11. Gasparri ML, Gentilini OD, Lueftner D, Kuehn T, Kaidar-Person O, Poortmans P. Changes in breast cancer management during the Corona Virus Disease 19 pandemic: 
An international survey of the European Breast Cancer Research Association of Surgical Trialists (EUBREAST). The Breast. 2020 08;52:110-115. https://doi.org/10.1016/j. breast.2020.05.006

12. Corsi F, Caruso A, Albasini S, Bossi D, Polizzi A, Piccotti F, Truffi M. Management of breast cancer in an EUSOMAaccredited Breast Unit in Lombardy, Italy, during the COVID-19 pandemic. The Breast Journal. 202005 30;. https://doi.org/10.1111/tbj.13926

13. de Azambuja E, Trapani D, Loibl S, Delaloge S, Senkus E, Criscitiello C, Poortman P, Gnant M, Di Cosimo S, Cortes J, Cardoso F, Paluch-Shimon S, Curigliano G. ESMO Management and treatment adapted recommendations in the COVID-19 era: Breast Cancer. ESMO Open. 2020 05;5(Supp1 3):e000793. https://doi.org/10.1136/ esmoopen-2020-000793

14. Vuagnat P, , Frelaut M, Ramtohul T, Basse C, Diakite S, Noret A, Bellesoeur A, Servois V, Hequet D, Laas E, Kirova Y, Cabel L, Pierga J, Bozec L, Paoletti X, Cottu P, Bidard F. COVID-19 in breast cancer patients: a cohort at the Institut Curie hospitals in the Paris area. Breast Cancer Research. 202005 28;22(1). https://doi.org/10.1186/s13058020-01293-8

15. Desideri I, Pilleron S, Battisti NML, Gomes F, de Glas N, Neuendorff NR, Liposits G, Paredero-Pérez I, Lok WCW, Loh KP, DuMontier C, Mian H, Soto-Perez-deCelis E. Caring for older patients with cancer during the COVID-19 pandemic: A Young International Society of Geriatric Oncology (SIOG) global perspective. Journal of Geriatric Oncology. 2020 05; https://doi.org/10.1016/j. jgo.2020.05.001

16. Curigliano G, Cardoso MJ, Poortmans P, Gentilini O, Pravettoni G, Mazzocco K, Houssami N, Pagani O, Senkus E, Cardoso F. Recommendations for triage, prioritization and treatment of breast cancer patients during the COVID-19 pandemic. The Breast. 2020 08;52:8-16. https://doi. org/10.1016/j.breast.2020.04.006

17. Dietz JR, Moran MS, Isakoff SJ, Kurtzman SH, Willey SC, Burstein HJ, Bleicher RJ, Lyons JA, Sarantou T, Baron PL, Stevens RE, Boolbol SK, Anderson BO, Shulman LN, Gradishar WJ, Monticciolo DL, Plecha DM, Nelson H, Yao KA. Recommendations for prioritization, treatment, and triage of breast cancer patients during the COVID-19 pandemic. the COVID-19 pandemic breast cancer consortium. Breast Cancer Research and Treatment. 2020 04 24;181(3):487-497. https://doi.org/10.1007/s10549020-05644-z

18. Soran A, Gimbel M, Diego E. Breast Cancer Diagnosis, Treatment and Follow-Up During COVID-19 Pandemic. European Journal of Breast Health. 202004 06;16(2):86-88. https://doi.org/10.5152/ejbh.2020.240320

\section{(i) (8)}

This work is licensed under a Creative Commons AttributionNon Commercial 4.0 International License. 